\title{
Corrigendum to 'The role of molecule-surface interaction in thermophoresis of an aerosol particle' [Journal of Aerosol Science, Volume 128, February 2019, Pages 62-71]
}

\author{
V.G. Chernyak*, T.V. Sograbi \\ Institute of Natural Sciences and Mathematics, Ural Federal University, 620083, Ekaterinburg, Russia
}

Authors regret to inform that a mistake was made in the indicated article when writing the expression (28) for the thermophoretic force. Hence incorrect expressions are obtained for the velocity of thermophoresis $(33,38,42,45$, 53). Besides this, in the preexponential factor of the expression (47) for the CL-model of scattering kernel $c_{r}$ should be changed to $\left|c_{r}^{\prime}\right|$.

The expression (28) for the thermophoretic force should be written as follows:

$$
\mathbf{F}_{T}=-\frac{16}{15} \sqrt{\pi} r_{0}^{2} \frac{\lambda}{\bar{v}} \nabla T_{\infty}\left[1-\frac{2}{\pi}\left\{\left(c_{r}^{2}, \widehat{K}\left[c_{r}\left(c^{\prime 2}-\frac{5}{2}\right)\right]\right)+2\left(c_{r} c_{\theta}, \widehat{K}\left[c_{\theta}\left(c^{2}-\frac{5}{2}\right)\right]\right)\right\}\right]
$$

All numerical coefficients in expressions (32), (41), (44) for the thermophoretic force $\mathbf{F}_{T}$ and (33), (42), (45) for the thermophoretic velocity $\mathbf{U}_{T}$ should be two times less. The expressions for the thermophoretic force (37) and velocity (38) should be written as follows:

$$
\mathbf{F}_{T}=-\frac{16}{15} \sqrt{\pi} r_{0}^{2} \frac{\lambda}{\bar{v}} \nabla T_{\infty}\left[1+\frac{5 \pi}{8} \frac{A K_{T}}{\chi}\right] ; \mathbf{U}_{T}=-\frac{1}{5} \frac{\lambda \nabla T_{\infty}}{p_{0}} \frac{8 \chi+5 \pi A K_{T}}{8 \chi+A \pi(K d+\chi)} .
$$

The expressions (52) and (53) are:

$$
\begin{aligned}
& \mathbf{F}_{T}=-\frac{16}{15} \sqrt{\pi} r_{0}^{2} \frac{\lambda}{\bar{v}} \nabla T_{\infty}\left[1-\frac{1}{2}\left(1-\alpha_{\tau}\right)+\frac{3 \pi}{16}\left(1-\sigma_{n}\right)\right] \\
& \mathbf{U}_{T}=-\frac{1}{5} \frac{\lambda \nabla T_{\infty}}{p_{0}} \frac{8}{8+\pi}\left[1+\frac{\pi}{4}\left(1-\sigma_{n}\right)\left(\frac{3}{4}-\frac{1}{8+\pi}\right)-\left(1-\alpha_{\tau}\right)\left(\frac{1}{2}-\frac{4}{8+\pi}\right)\right] .
\end{aligned}
$$

Therefore, it is necessary to correct some of the graphs given in the article.

The correct form is:

DOI of original article: https://doi.org/10.1016/j.jaerosci.2018.11.012.

* Corresponding author.

E-mail address: vladimir.chernyak@urfu.ru (V.G. Chernyak). 

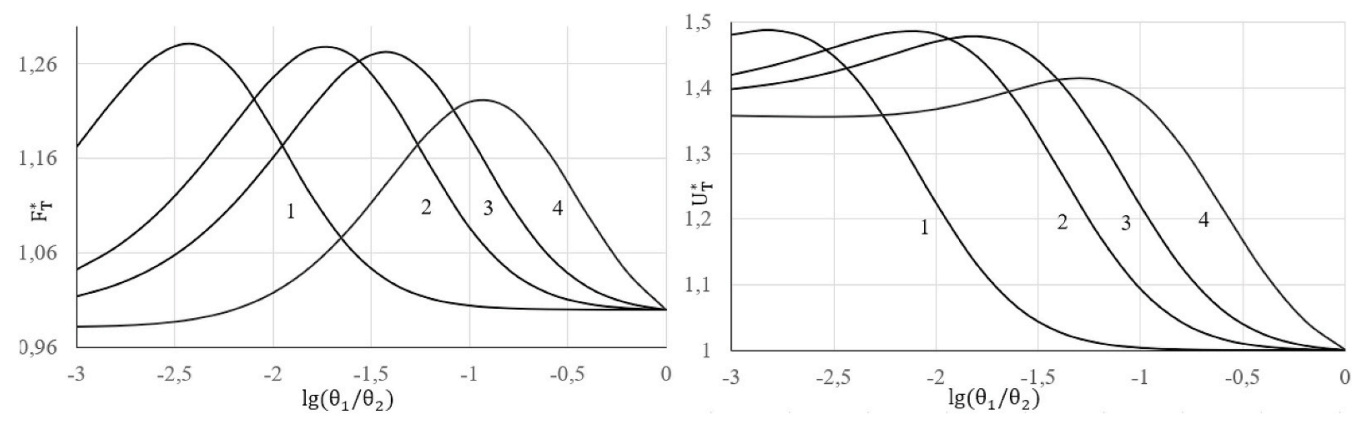

Fig. 2.

For example, at $\theta_{1} / T_{0}=0.01 \div 0.1$ and $\varepsilon_{\infty}=1$, the maximum excess is $28 \%$.
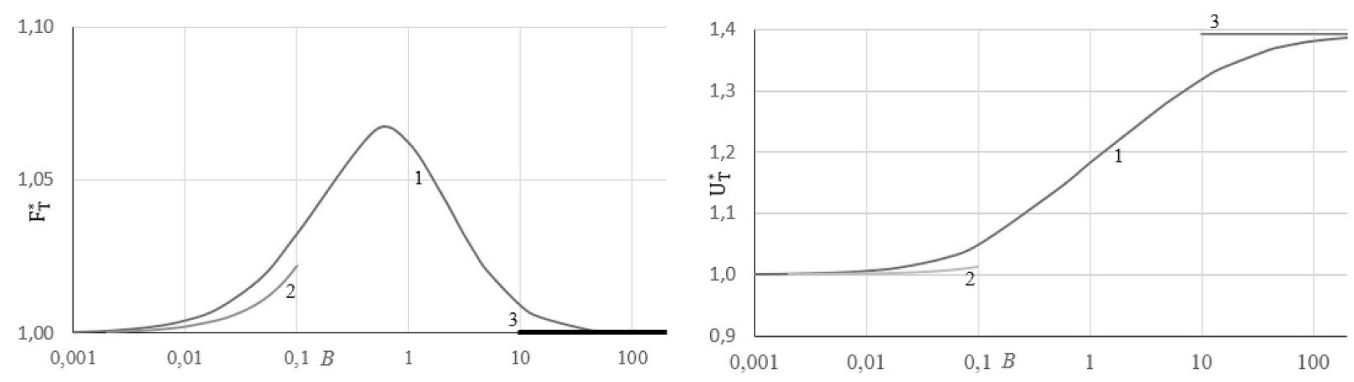

Fig. 3.

At intermediate values of $B$, the thermophoretic force has a maximum that exceeds by $8 \%$ the value of the force with complete accommodation.
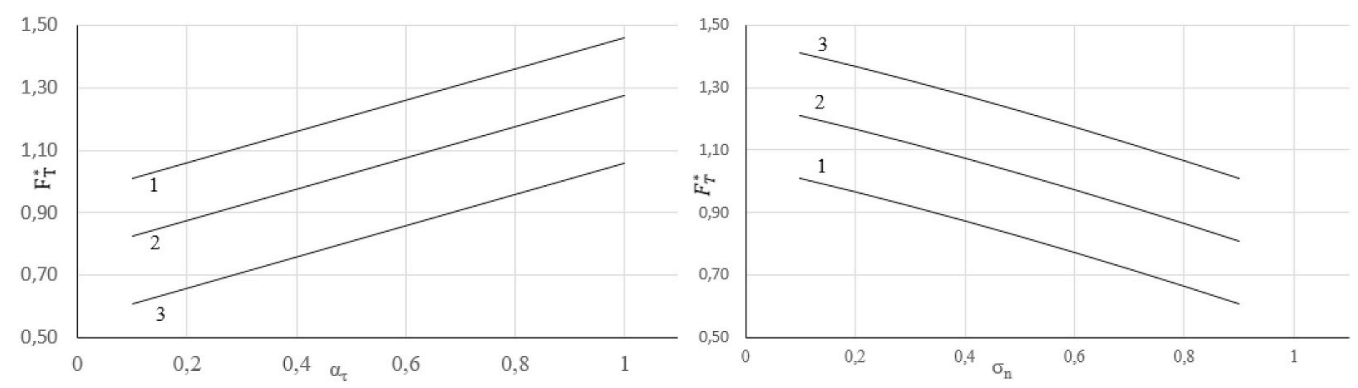

Fig. 4.

The Conclusion section contains an erroneous statement « In the case of the CL-model, when the accommodation parameters are zero, this force is zero».

The authors would like to apologise for any inconvenience caused. 